\title{
$S$-shaped connected component for the fourth-order boundary value problem
}

Jinxiang Wang ${ }^{1,2}$ and and Ruyun Ma ${ }^{1 *}$

${ }^{\text {*Correspondence: }}$ mary@nwnu.edu.cn 'Department of Mathematics, Northwest Normal University, Lanzhou, 730070, P.R. China Full list of author information is available at the end of the article
C 2016 Wang and Ma. This article is distributed under the terms of the Creative Commons Attribution 4.0 International License (http://creativecommons.org/licenses/by/4.0/), which permits unrestricted use, distribution, and reproduction in any medium, provided you give appropriate credit to the original author(s) and the source, provide a link to the Creative Commons license, and indicate if changes were made.

\section{Introduction}

The fourth-order boundary value problem

$$
\left\{\begin{array}{l}
u^{\prime \prime \prime \prime}(x)=\lambda f\left(x, u(x), u^{\prime \prime}(x)\right), \quad x \in(0,1) \\
u(0)=u(1)=u^{\prime \prime}(0)=u^{\prime \prime}(1)=0
\end{array}\right.
$$

describes the deformations of an elastic beam with ends simply supported at 0 and 1; see Gupta [1], Lazer and McKenna [2], and references therein. The solvability of (1.1) with $\lambda \equiv 1$ has been extensively studied by several authors under the at most linear growth conditions; see Usmani [3], Yang [4], Del Pino [5], and Cabada [6]. However, these results give no information about the sign of solutions.

The positivity (or negativity) of solutions of beam equation (i.e., the deflection of the roadbed) is crucial for the whole system and properties of possibly nonstationary solutions. Even if we focus on one-dimensional ODE problems, we would like to mention works of Grunau and Sweers [7, 8], where positive solutions of fourth-order PDEs subject to different types of boundary conditions are investigated. The existence and multiplicity of positive solutions of (1.1) or its particular case have been investigated via theory of fixed point index in a cone and Leray-Schauder degree by many authors; see, for example, 
[9-17] and the references therein. Notice that these results give no information on the interesting problem as to what happens to the norms of positive solutions of (1.1) as $\lambda$ varies in $\mathbb{R}^{+}$.

Recently, the global behavior of solution set of (1.1) has been studied by using Dancer's or Rabinowitz's global bifurcation theorem (see Ma [18], Ma, Gao, and Han [19], and Dai and Han [20]), and accordingly, the existence and multiplicity of positive solutions and nodal solutions have been obtained. However, the sublinear and superlinear conditions or asymptotic linear growth conditions imposed on the nonlinearities only deduce a relatively simple "shape of the component."

For the second-order boundary value problem

$$
\left\{\begin{array}{l}
y^{\prime \prime}(x)=\lambda f(x, y(x)), \quad x \in(0,1), \\
y(0)=y(1)=0
\end{array}\right.
$$

there have been some results on the existence of an $S$-shaped component. For example, in the recent paper [21], Kim and Tanaka showed the existence of three positive solutions via proving the existence of an $S$-shaped connected component in the solution set of the following problem:

$$
\left\{\begin{array}{l}
\left(\left|y^{\prime}\right|^{p-2} y^{\prime}\right)^{\prime}=\lambda a(x) f(y), \quad x \in(0,1) \\
y(0)=y(1)=0
\end{array}\right.
$$

More precisely, they prove the following:

Theorem A ([21], Theorem 1.1) Assume that the following conditions hold:

(F1) there exist $x_{1}, x_{2} \in[0,1]$ such that $x_{1}<x_{2}, a(x)>0$ on $\left(x_{1}, x_{2}\right)$, and $a(x) \leq 0$ on $[0,1] \backslash\left[x_{1}, x_{2}\right]$;

(F2) there exist $\alpha>0, f_{0}>0$, and $f_{1}>0$ such that $\lim _{s \rightarrow 0^{+}} \frac{f(s)-f_{0} s^{p-1}}{s^{p-1+\alpha}}=-f_{1}$;

(F3) $f_{\infty}:=\lim _{s \rightarrow \infty} \frac{f(s)}{s^{p-1}}=0$;

(F4) there exists $s_{0}>0$ such that

$$
\min _{s \in\left[s_{0}, 2 s_{0}\right]} \frac{f(s)}{s^{p-1}} \geq \frac{f_{0}(p-1)}{\mu_{1} h_{0}}\left(\frac{\pi_{p}}{x_{2}-x_{1}}\right)^{p}
$$

where $\mu_{1}>0$ is the simple principal eigenvalue of the linear problem corresponding to (1.3), and

$$
\pi_{p}:=\frac{2 \pi}{p \sin \left(\frac{\pi}{p}\right)}, \quad h_{0}=\min _{x \in\left[\frac{3 x_{1}+x_{2}}{4}, \frac{x_{1}+3 x_{2}}{4}\right]} h(x) .
$$

Then there exist $\lambda_{*} \in\left(0, \frac{\mu_{1}}{f_{0}}\right)$ and $\lambda^{*}>\frac{\mu_{1}}{f_{0}}$ such that

(i) (1.3) has at least one positive solution if $\lambda=\lambda_{*}$;

(ii) (1.3) has at least two positive solutions if $\lambda_{*}<\lambda \leq \frac{\mu_{1}}{f_{0}}$;

(iii) (1.3) has at least three positive solutions if $\frac{\mu_{1}}{f_{0}}<\lambda<\lambda^{*}$;

(iv) (1.3) has at least two positive solutions if $\lambda=\lambda^{*}$;

(v) (1.3) has at least one positive solution if $\lambda>\lambda^{*}$. 
Of course, the natural question is what happens if we consider the fourth-order problem (1.1)? As we know, there are great differences between second-order and fourth-order BVPs; for example, for second-order BVPs, the existence of a well-ordered pair of lower and upper solutions is sufficient to ensure the existence of a solution enclosed by them; see [22]. But this is not correct for fourth-order BVPs even for the simple boundary conditions, as the authors showed in [6]. On the other hand, the concavity and convexity of the solutions of second-order BVPs can be deduced directly from the nonlinearity in the equation, but for fourth-order BVPs, this becomes complicated, especially when the nonlinearity changes the sign.

The purpose of this paper is to investigate the existence of an $S$-shaped component in the solution set of problem (1.1).

Let

$$
\mathcal{C}=\left\{\left(\lambda, u_{\lambda}\right): \lambda>0 \text { and } u_{\lambda} \text { is a solution of }(1.1)\right\}
$$

be a component in the solution set of problem (1.1). On the $\left(\lambda,\left\|u_{\lambda}\right\|_{\infty}\right)$-plane, we define the component curve of $\mathcal{C}$ as follows:

$$
\widetilde{\mathcal{C}}=\left\{\left(\lambda,\left\|u_{\lambda}\right\|_{\infty}\right):\left(\lambda, u_{\lambda}\right) \in \mathcal{C}\right\} .
$$

We say that $\mathcal{C}$ is $S$-shaped if $\widetilde{\mathcal{C}}$ has at least two turning points at some points $\left(\lambda^{*},\left\|u_{\lambda^{*}}\right\|_{\infty}\right)$ and $\left(\lambda_{*},\left\|u_{\lambda_{*}}\right\|_{\infty}\right)$, where $\lambda_{*}<\lambda^{*}$ are two positive numbers such that

(i) $\left\|u_{\lambda^{*}}\right\|_{\infty}<\left\|u_{\lambda_{*}}\right\|_{\infty}$,

(ii) at $\left(\lambda^{*},\left\|u_{\lambda^{*}}\right\|_{\infty}\right)$, the component curve $\widetilde{\mathcal{C}}$ turns to the left,

(iii) at $\left(\lambda_{*},\left\|u_{\lambda_{*}}\right\|_{\infty}\right)$, the component curve $\widetilde{\mathcal{C}}$ turns to the right.

As we mentioned before, the change of sign of $f$ brings a great difficulty to the solvability of (1.1), so in this paper, we only consider the nonnegative case. Evidently, if there exists an $S$-shaped component in the positive solutions set of problem (1.1), then we can accordingly deduce the existence and multiplicity of positive solutions for problem (1.1) and, especially, establish the existence of three distinct positive solutions for $\lambda$ being in a certain interval.

It is worth remarking that the existence of an $S$-shaped component has its independent interest. This relationship is very useful for computing the numerical solutions of (1.1) since it can be used to guide the numerical work. For example, it can be used to estimate the $u$-interval in advance in applying the finite difference method. Moreover, we note that if $f$ is nonnegative, then any positive solution $u$ of (1.1) satisfies

$$
\|u\|_{\infty}=u(\tau)=\int_{0}^{\tau} u^{\prime}(s) d s \leq \int_{0}^{1} u^{\prime}(0) d s=u^{\prime}(0),
$$

and this, together with the $S$-shaped connected component, can be used to restrict the range of initial values in applying the shooting method.

Throughout the paper, we assume that

(H1) $f:[0,1] \times[0, \infty) \times(-\infty, 0] \rightarrow[0, \infty)$ is continuous, and $f(x, s, p)>0$ for $x \in[0,1]$ and $(s, p) \in([0, \infty) \times(-\infty, 0]) \backslash\{(0,0)\}$

(H2) there exist constants $a, b \in[0, \infty)$ with $a+b>0$ and $c>0, d>0$ such that

$$
\lim _{\sqrt{s^{2}+p^{2}} \rightarrow 0} \frac{f(x, s, p)-(a s-b p)}{\sqrt{s^{2}+p^{2}}}=-d \quad \text { uniformly for } x \in[0,1] ;
$$


(H3)

$$
\lim _{\sqrt{s^{2}+p^{2}} \rightarrow \infty} \frac{f(x, s, p)}{\sqrt{s^{2}+p^{2}}}=0 \quad \text { uniformly for } x \in[0,1]
$$

(H4) there exists $s_{0}>0$ such that

$$
\min _{s \in\left[s_{0}, 4 s_{0}\right]} \frac{f(x, s, p)}{s} \geq \frac{16 \pi^{4}}{\lambda_{1}(a, b)} \quad \text { uniformly for } x \in[0,1], p \in(-\infty, 0]
$$

where $\lambda_{1}(a, b)>0$ is the generalized principal eigenvalue of the linear problem

$$
\left\{\begin{array}{l}
u^{\prime \prime \prime \prime}(x)=\lambda\left(a u-b u^{\prime \prime}\right), \quad x \in(0,1) \\
u(0)=u(1)=u^{\prime \prime}(0)=u^{\prime \prime}(1)=0
\end{array}\right.
$$

defined in Lemma 2.1.

It is easy to find that if $(\mathrm{H} 2)$ holds, then

$$
f(x, s, p)=a s-b p+\circ\left(\sqrt{s^{2}+p^{2}}\right) \quad \text { as } \sqrt{s^{2}+p^{2}} \rightarrow 0 .
$$

Moreover, if (1.5) and (H3) hold, then there exist constants $A, B \in[0, \infty)$ with $A+B>0$ such that

$$
f(x, s, p) \leq A s-B p \quad \text { uniformly for } x \in[0,1] .
$$

Considering the shape of a component in the positive solution set of problem (1.1), we have the following result.

Theorem 1.1 Assume that (H1), (H2), (H3), and (H4) hold. Then there exist $\lambda_{*} \in$ $\left(0, \lambda_{1}(a, b)\right)$ and $\lambda^{*}>\lambda_{1}(a, b)$ such that

(i) (1.1) has at least one positive solution if $\lambda=\lambda_{*}$;

(ii) (1.1) has at least two positive solutions if $\lambda_{*}<\lambda \leq \lambda_{1}(a, b)$;

(iii) (1.1) has at least three positive solutions if $\lambda_{1}(a, b)<\lambda<\lambda^{*}$;

(iv) (1.1) has at least two positive solutions if $\lambda=\lambda^{*}$;

(v) (1.1) has at least one positive solution if $\lambda>\lambda^{*}$.

Remark 1.1 Using the Amann's three-solution theorem, Cabada et al. [6] proved the existence of at least three solutions in the presence of lower and upper solutions for the fourth-order problem

$$
\left\{\begin{array}{l}
u^{\prime \prime \prime \prime}(x)=f(x, u(x)), \quad x \in(0,1), \\
u(0)=u(1)=u^{\prime \prime}(0)=u^{\prime \prime}(1)=0,
\end{array}\right.
$$

where $f$ is bounded and satisfies the unilateral Lipschitz condition. Using the Morse theory, Han and $\mathrm{Xu}$ [23] obtained an existence theorem on three solutions of problem (1.7), where $f(x, u)$ is differentiable in $[0,1] \times \mathbb{R}$. Our conditions imposed here are quite different from those in [6] and [23]. Clearly, problem (1.7) can be seen as a particular case of (1.1), and thus Theorem 1.1 extend the results of [6] and [23]. 
The rest of this paper is arranged as follows. In Section 2, we show a global bifurcation phenomena from the trivial branch with rightward direction. Section 3 is devoted to show that there are at least two direction turns of the component and complete the proof of Theorem 1.1.

\section{Rightward bifurcation}

In this section, we state some preliminary results and show a global bifurcation phenomenon from the trivial branch with rightward direction.

Definition 2.1 Let $\alpha, \beta \in[0, \infty)$ be given constants with $\alpha+\beta>0$. We say that $\lambda$ is a generalized eigenvalue of the linear problem

$$
\left\{\begin{array}{l}
u^{\prime \prime \prime \prime}(x)=\lambda\left(\alpha u-\beta u^{\prime \prime}\right), \quad x \in(0,1), \\
u(0)=u(1)=u^{\prime \prime}(0)=u^{\prime \prime}(1)=0,
\end{array}\right.
$$

if (2.1) has a nontrivial solution.

Lemma 2.1 ([18], Theorem 3.1) Let $\alpha, \beta \in[0, \infty)$ be given constants with $\alpha+\beta>0$. Then the generalized eigenvalues of (2.1) are given by

$$
\lambda_{1}(\alpha, \beta)<\lambda_{2}(\alpha, \beta)<\cdots<\lambda_{n}(\alpha, \beta)<\cdots,
$$

where

$$
\lambda_{k}(\alpha, \beta)=\frac{(k \pi)^{4}}{\alpha+\beta(k \pi)^{2}}, \quad k \in \mathbb{N} .
$$

The generalized eigenfunction corresponding to $\lambda_{k}(\alpha, \beta)$ is $\varphi_{k}(t)=\sin k \pi t$.

Remark 2.1 The first generalized eigenvalue $\lambda_{1}(\alpha, \beta)$ of the linear problem (2.1) is the minimum of the Rayleigh quotient, that is,

$$
\begin{aligned}
\lambda_{1}(\alpha, \beta)= & \inf \left\{\frac{\int_{0}^{1}\left(u^{\prime \prime}(x)\right)^{2} d x}{\int_{0}^{1}\left(\alpha u^{2}(x)+\beta\left(u^{\prime}(x)\right)^{2}\right) d x} \mid u \in C^{4}[0,1], u \neq \equiv 0\right. \\
& \text { and } \left.u(0)=u(1)=u^{\prime \prime}(0)=u^{\prime \prime}(1)=0\right\} .
\end{aligned}
$$

Moreover, the corresponding eigenfunction $\varphi_{1}(t)=\sin \pi t$ is positive in $(0,1)$.

Let $g \in C[0,1]$. It is well known that the fourth-order linear problem

$$
\left\{\begin{array}{l}
v^{\prime \prime \prime \prime}=g(t), \quad t \in(0,1), \\
v(0)=v(1)=v^{\prime \prime}(0)=v^{\prime \prime}(1)=0
\end{array}\right.
$$

has a unique solution

$$
v(t)=\int_{0}^{1} \int_{0}^{1} G(t, s) G(s, \tau) g(\tau) d \tau d s
$$


where

$$
G(t, s)= \begin{cases}s(1-t), & 0 \leq s \leq t \leq 1 \\ t(1-s), & 0 \leq t \leq s \leq 1\end{cases}
$$

Moreover, if $g \geq 0$ and $g \not \equiv 0$, then

$$
v^{\prime \prime}(t)=-\int_{0}^{1} G(t, s) g(s) d s \leq 0,
$$

that is, $v \geq 0$ is concave.

The Green's function $G(t, s)$ has the following properties:

(i) $0 \leq G(t, t) G(s, s) \leq G(t, s) \leq G(s, s), \forall t, s \in(0,1)$;

(ii) $G(t, s) \geq \frac{1}{4} G(s, s), \forall t \in\left[\frac{1}{4}, \frac{3}{4}\right], s \in(0,1)$.

Therefore, for any $t \in\left[\frac{1}{4}, \frac{3}{4}\right]$, we have

$$
\begin{aligned}
v(t) & =\int_{0}^{1} G(t, s)\left[\int_{0}^{1} G(s, \tau) g(\tau) d \tau\right] d s \\
& \geq \frac{1}{4} \int_{0}^{1} G(s, s)\left[\int_{0}^{1} G(s, \tau) g(\tau) d \tau\right] d s \\
& \geq \frac{1}{4}\|v\|_{\infty} .
\end{aligned}
$$

Let

$$
e(x):=\sin \pi x, \quad x \in[0,1]
$$

and

$$
\begin{aligned}
X= & \left\{u \in C^{2}[0,1] \mid u(0)=u(1)=u^{\prime \prime}(0)=u^{\prime \prime}(1)=0,\right. \\
& \left.\exists \gamma \in(0, \infty), \text { s.t. }-\gamma e(x) \leq u^{\prime \prime}(x) \leq \gamma e(x) \text { for } x \in[0,1]\right\} .
\end{aligned}
$$

We define the norm of $X$ by

$$
\|u\|_{X}:=\inf \left\{\gamma \mid-\gamma e(x) \leq u^{\prime \prime}(x) \leq \gamma e(x), x \in[0,1]\right\} .
$$

It is easy to check that $\left(X,\|\cdot\|_{X}\right)$ is a Banach space and

$$
\|u\|_{\infty} \leq\left\|u^{\prime \prime}\right\|_{\infty} \leq\|u\|_{X}
$$

Let

$$
P:=\left\{u \in X \mid u^{\prime \prime}(x) \leq 0, u(x) \geq 0, x \in[0,1]\right\} .
$$

Then $P$ is a normal cone of $X$ and has a nonempty interior, and $X=\overline{P-P}$. Let $\zeta \in C([0,1] \times[0, \infty) \times(-\infty, 0])$ be such that

$$
f(x, s, p)=a s-b p+\zeta(x, s, p)
$$


Clearly, (1.5) implies that

$$
\lim _{\sqrt{s^{2}+p^{2}} \rightarrow 0} \frac{\zeta(x, s, p)}{\sqrt{s^{2}+p^{2}}}=0 \quad \text { uniformly for } x \in[0,1] .
$$

Then we consider

$$
\left\{\begin{array}{l}
u^{\prime \prime \prime \prime}(x)=\lambda\left(a u-b u^{\prime \prime}\right)+\lambda \zeta\left(x, u, u^{\prime \prime}\right), \quad x \in(0,1) \\
u(0)=u(1)=u^{\prime \prime}(0)=u^{\prime \prime}(1)=0
\end{array}\right.
$$

as a bifurcation problem from the trivial solution $u \equiv 0$.

Using the Dancer bifurcation theorem and following the arguments in the proof of Theorem 4.1 in [18], we have the following:

Lemma 2.2 ([18]) Assume that $(\mathrm{H} 1)$ and $(\mathrm{H} 2)$ hold. Then from $\left(\lambda_{1}(a, b), 0\right)$ there emanate an unbounded subcontinuum $\mathcal{C}$ of positive solutions of (1.1) in the set

$$
\{(\lambda, u) \in(0, \infty) \times P: u \in \operatorname{int} P\}
$$

Lemma 2.3 Assume that (H1), (H2), and (H3) hold. Let $\left\{\left(\lambda_{n}, u_{n}\right)\right\}$ be a sequence of positive solutions to (1.1) that satisfies $\lambda_{n} \rightarrow \lambda_{1}(a, b)$ and $\left\|u_{n}\right\|_{X} \rightarrow 0$. Then there exists a subsequence of $\left\{u_{n}\right\}$, again denoted by $\left\{u_{n}\right\}$, such that $\frac{u_{n}}{\left\|u_{n}\right\|_{X}}$ converges uniformly to $\frac{\sin \pi x}{\pi^{2}}$ on $[0,1]$.

Proof Set $v_{n}:=\frac{u_{n}}{\left\|u_{n}\right\|_{X}}$. Then $\left\|v_{n}\right\|_{X}=1$, and (2.3) implies that $\left\|v_{n}\right\|_{\infty}$ and $\left\|v_{n}^{\prime \prime}\right\|_{\infty}$ are bounded. By the Ascoli-Arzelà theorem a subsequence of $v_{n}$ uniformly converges to a limit $v$, and we again denote by $v_{n}$ the subsequence.

For every $\left(\lambda_{n}, u_{n}\right)$, we have

$$
u_{n}(x)=\lambda_{n} \int_{0}^{1} G(x, s)\left[\int_{0}^{1} G(s, \tau) f\left(\tau, u_{n}(\tau), u_{n}^{\prime \prime}(\tau)\right) d \tau\right] d s
$$

Dividing both sides of (2.4) by $\left\|u_{n}\right\|_{X}$, we get

$$
v_{n}(x)=\lambda_{n} \int_{0}^{1} G(x, s)\left[\int_{0}^{1} G(s, \tau) \frac{f\left(\tau, u_{n}(\tau), u_{n}^{\prime \prime}(\tau)\right)}{\left\|u_{n}\right\|_{X}} d \tau\right] d s .
$$

Combining (1.6) with (2.3), we have

$$
\frac{f\left(\tau, u_{n}(\tau), u_{n}^{\prime \prime}(\tau)\right)}{\left\|u_{n}\right\|_{X}} \leq \frac{A u_{n}(\tau)-B u_{n}^{\prime \prime}(\tau)}{\left\|u_{n}\right\|_{X}} \leq A+B \quad \text { uniformly for } \tau \in[0,1] .
$$

Recalling (1.5), Lebesgue's dominated convergence theorem shows that

$$
v(x)=\lambda_{1}(a, b) \int_{0}^{1} G(x, s)\left[\int_{0}^{1} G(s, \tau)\left(a v(\tau)-b v^{\prime \prime}(\tau)\right) d \tau\right] d s,
$$

which means that $v$ is a nontrivial solution of (2.1) with $\lambda=\lambda_{1}(a, b)$, and hence there exists a constant $k$ such that $v \equiv k \varphi_{1}=k \sin \pi x$ since $\|v\|_{X}=1$, and then $k=\frac{1}{\pi^{2}}$. 
Remark 2.2 From the proof of Lemma 2.3 we have that $\frac{u_{n}^{\prime \prime}}{\left\|u_{n}\right\|_{X}}$ converges uniformly to $-\sin \pi x$ on $[0,1]$.

Lemma 2.4 Assume that (H1), (H2), and (H3) hold. Let $\mathcal{C}$ be as in Lemma 2.2. Then there exists $\delta>0$ such that $(\lambda, u) \in \mathcal{C}$ and $\left|\lambda-\lambda_{1}(a, b)\right|+\|u\|_{X} \leq \delta$ imply $\lambda>\lambda_{1}(a, b)$.

Proof Assume to the contrary that there exists a sequence $\left\{\left(\lambda_{n}, u_{n}\right)\right\} \subset \mathcal{C}$ such that $\lambda_{n} \rightarrow$ $\lambda_{1}(a, b),\left\|u_{n}\right\|_{X} \rightarrow 0$, and $\lambda_{n} \leq \lambda_{1}(a, b)$. By Lemma 2.3 there exists a subsequence of $\left\{u_{n}\right\}$, again denoted by $\left\{u_{n}\right\}$, such that $\frac{u_{n}}{\left\|u_{n}\right\|_{X}}$ converges uniformly to $\frac{\sin \pi x}{\pi^{2}}$ and $\frac{u_{n}^{\prime \prime}}{\left\|u_{n}\right\|_{X}}$ converges uniformly to $-\sin \pi x$ on $[0,1]$. Multiplying the equation of (1.1) with $(\lambda, u)=\left(\lambda_{n}, u_{n}\right)$ by $u_{n}$ and integrating over $[0,1]$, we have

$$
\int_{0}^{1} u_{n}(x) u_{n}^{\prime \prime \prime \prime}(x) d x=\lambda_{n} \int_{0}^{1} f\left(x, u_{n}(x), u_{n}^{\prime \prime}(x)\right) u_{n}(x) d x
$$

By simple computation and using the definition of $\lambda_{1}(a, b)$ in Remark 2.1, we get

$$
\begin{aligned}
\int_{0}^{1} u_{n}(x) u_{n}^{\prime \prime \prime \prime}(x) d x & =\int_{0}^{1}\left(u_{n}^{\prime \prime}(x)\right)^{2} d x \\
& \geq \lambda_{1}(a, b) \int_{0}^{1}\left(a u_{n}^{2}(x)+b\left(u_{n}^{\prime}(x)\right)^{2}\right) d x
\end{aligned}
$$

Combining (2.8) with (2.9), we have

$$
\begin{aligned}
\int_{0}^{1} f\left(x, u_{n}, u_{n}^{\prime \prime}\right) u_{n} d x & \geq \frac{\lambda_{1}(a, b)}{\lambda_{n}} \int_{0}^{1}\left(a u_{n}^{2}(x)+b\left(u_{n}^{\prime}(x)\right)^{2}\right) d x \\
& =\frac{\lambda_{1}(a, b)}{\lambda_{n}} \int_{0}^{1}\left(a u_{n}(x)-b u_{n}^{\prime \prime}(x)\right) u_{n}(x) d x
\end{aligned}
$$

that is,

$$
\begin{gathered}
\frac{\int_{0}^{1} f\left(x, u_{n}, u_{n}^{\prime \prime}\right) u_{n} d x-\int_{0}^{1}\left(a u_{n}(x)-b u_{n}^{\prime \prime}(x)\right) u_{n}(x) d x}{\left\|u_{n}\right\|_{X}^{2+c}} \\
\geq \frac{\left(\frac{\lambda_{1}(a, b)}{\lambda_{n}}-1\right) \int_{0}^{1}\left(a u_{n}(x)-b u_{n}^{\prime \prime}(x)\right) u_{n}(x) d x}{\left\|u_{n}\right\|_{X}^{2+c}} .
\end{gathered}
$$

Since

$$
\begin{aligned}
& \frac{\int_{0}^{1} f\left(x, u_{n}, u_{n}^{\prime \prime}\right) u_{n} d x-\int_{0}^{1}\left(a u_{n}(x)-b u_{n}^{\prime \prime}(x)\right) u_{n}(x) d x}{\left\|u_{n}\right\|_{X}^{2+c}} \\
& =\int_{0}^{1} \frac{\left[f\left(x, u_{n}, u_{n}^{\prime \prime}\right)-\left(a u_{n}(x)-b u_{n}^{\prime \prime}(x)\right)\right] u_{n}(x) \frac{\sqrt{u_{n}^{2}+u_{n}^{\prime \prime 2}}}{\left\|u_{n}\right\|_{X}^{2+c}} d x}{{\sqrt{u_{n}^{2}+u_{n}^{\prime 2}}}^{1+c}} d x \\
& =\int_{0}^{1} \frac{\left[f\left(x, u_{n}, u_{n}^{\prime \prime}\right)-\left(a u_{n}(x)-b u_{n}^{\prime \prime}(x)\right)\right]}{{\sqrt{u_{n}^{2}+u_{n}^{\prime \prime 2}}}^{1+c}} \frac{u_{n}(x)}{\left\|u_{n}\right\|_{X}}\left(\frac{u_{n}^{2}+u_{n}^{\prime \prime 2}}{\left\|u_{n}\right\|_{X}^{2}}\right)^{\frac{1+c}{2}} d x,
\end{aligned}
$$


Lebesgue's dominated convergence theorem, (2.3), condition (H2), and Lemma 2.3 imply that

$$
\begin{array}{r}
\frac{\int_{0}^{1} f\left(x, u_{n}, u_{n}^{\prime \prime}\right) u_{n} d x-\int_{0}^{1}\left(a u_{n}(x)-b u_{n}^{\prime \prime}(x)\right) u_{n}(x) d x}{\left\|u_{n}\right\|_{X}^{2+c}} \\
\rightarrow-d \int_{0}^{1} \frac{\sin \pi x}{\pi^{2}}\left(\frac{\sin ^{2} \pi x}{\pi^{4}}+\sin ^{2} \pi x\right)^{\frac{1+c}{2}} d x<0 .
\end{array}
$$

Similarly,

$$
\begin{aligned}
& \frac{\left(\frac{\lambda_{1}(a, b)}{\lambda_{n}}-1\right) \int_{0}^{1}\left(a u_{n}(x)-b u_{n}^{\prime \prime}(x)\right) u_{n}(x) d x}{\left\|u_{n}\right\|_{X}^{2+c}} \\
& \quad=\left(\frac{\lambda_{1}(a, b)}{\lambda_{n}}-1\right) \frac{1}{\left\|u_{n}\right\|_{X}^{c}} \int_{0}^{1} \frac{u_{n}(x)}{\left\|u_{n}\right\|_{X}}\left(a \frac{u_{n}(x)}{\left\|u_{n}\right\|_{X}}-b \frac{u_{n}^{\prime \prime}(x)}{\left\|u_{n}\right\|_{X}}\right) d x,
\end{aligned}
$$

and by Lebesgue's dominated convergence theorem, (2.3), and Lemma 2.3 we have

$$
\begin{aligned}
& \int_{0}^{1} \frac{u_{n}(x)}{\left\|u_{n}\right\|_{X}}\left(a \frac{u_{n}(x)}{\left\|u_{n}\right\|_{X}}-b \frac{u_{n}^{\prime \prime}(x)}{\left\|u_{n}\right\|_{X}}\right) d x \\
& \quad \rightarrow \int_{0}^{1} \frac{\sin \pi x}{\pi^{2}}\left(a \frac{\sin \pi x}{\pi^{2}}+b \sin \pi x\right) d x>0
\end{aligned}
$$

which contradicts (2.11).

\section{Direction turns of component and proof of Theorem 1.1}

In this section, we show that there are at least two direction turns of the component under conditions (H3) and (H4), that is, the component is $S$-shaped, and accordingly, we finish the proof of Theorem 1.1.

Lemma 3.1 Assume that (H1) and (H4) hold. Let $u$ be a positive solution of (1.1) with $\|u\|_{\infty}=4 s_{0}$. Then $\lambda<\lambda_{1}(a, b)$.

Proof Let $u$ be a solution of (1.1) with $\|u\|_{\infty}=4 s_{0}$. Then (2.2) imply that

$$
\frac{1}{4}\|u\|_{\infty}=s_{0} \leq u(x) \leq\|u\|_{\infty}=4 s_{0}, \quad x \in\left[\frac{1}{4}, \frac{3}{4}\right] .
$$

Suppose on the contrary that $\lambda \geq \lambda_{1}(a, b)$. Then by (H4) we have

$$
\begin{aligned}
u^{\prime \prime \prime \prime}(x) & =\lambda f\left(x, u(x), u^{\prime \prime}(x)\right) \\
& \geq \lambda \frac{f\left(x, u(x), u^{\prime \prime}(x)\right)}{u(x)} u(x) \\
& \geq \lambda_{1}(a, b) \frac{16 \pi^{4}}{\lambda_{1}(a, b)} u(x) \\
& =16 \pi^{4} u(x), \quad x \in\left[\frac{1}{4}, \frac{3}{4}\right] .
\end{aligned}
$$


Multiplying inequality $(3.1)$ by $\sin \left[2 \pi\left(x-\frac{1}{4}\right)\right]$ and integrating over $\left[\frac{1}{4}, \frac{3}{4}\right]$, we have

$$
\int_{\frac{1}{4}}^{\frac{3}{4}} u^{\prime \prime \prime \prime} \sin \left[2 \pi\left(x-\frac{1}{4}\right)\right] d x \geq \int_{\frac{1}{4}}^{\frac{3}{4}} 16 \pi^{4} u(x) \sin \left[2 \pi\left(x-\frac{1}{4}\right)\right] d x
$$

On the other hand, by simple computation we have that

$$
\begin{aligned}
\int_{\frac{1}{4}}^{\frac{3}{4}} u^{\prime \prime \prime \prime} \sin \left[2 \pi\left(x-\frac{1}{4}\right)\right] d x \\
=2 \pi\left[u^{\prime \prime}\left(\frac{1}{4}\right)+u^{\prime \prime}\left(\frac{3}{4}\right)\right]-8 \pi^{3}\left[u\left(\frac{1}{4}\right)+u\left(\frac{3}{4}\right)\right] \\
+\int_{\frac{1}{4}}^{\frac{3}{4}} 16 \pi^{4} u(x) \sin \left[2 \pi\left(x-\frac{1}{4}\right)\right] d x
\end{aligned}
$$

Since $u(t) \geq 0$ is concave, we have $2 \pi\left[u^{\prime \prime}\left(\frac{1}{4}\right)+u^{\prime \prime}\left(\frac{3}{4}\right)\right]-8 \pi^{3}\left[u\left(\frac{1}{4}\right)+u\left(\frac{3}{4}\right)\right]<0$, which gives a contradiction.

Lemma 3.2 Assume that (H1), (H2), and (H3) hold. Then, $\mathcal{C}$ joins $\left(\lambda_{1}(a, b), 0\right)$ to $(\infty, \infty)$ in $[0, \infty) \times P$.

Proof We divide the proof into two steps.

Step 1 . We show that $\sup \{\lambda \mid(\lambda, u) \in \mathcal{C}\}=\infty$.

Assume on the contrary that $\sup \{\lambda \mid(\lambda, u) \in \mathcal{C}\}=: c_{0}<\infty$. Let $\left\{\left(\lambda_{n}, u_{n}\right)\right\} \subset \mathcal{C}$ be such that $\left|\lambda_{n}\right|+\left\|u_{n}\right\|_{X} \rightarrow \infty$. Then $\left\|u_{n}\right\|_{X} \rightarrow \infty$. Since $\left(\lambda_{n}, u_{n}\right) \in \mathcal{C}$, we have that

$$
\left\{\begin{array}{l}
u_{n}^{\prime \prime \prime \prime}(x)=\lambda_{n} f\left(x, u_{n}(x), u_{n}^{\prime \prime}(x)\right), \quad x \in(0,1) \\
u_{n}(0)=u_{n}(1)=u_{n}^{\prime \prime}(0)=u_{n}^{\prime \prime}(1)=0
\end{array}\right.
$$

Set $\omega_{n}:=\frac{u_{n}}{\left\|u_{n}\right\|_{X}}$. Then $\left\|\omega_{n}\right\|_{X}=1$, and

$$
\left\{\begin{array}{l}
\omega_{n}^{\prime \prime \prime \prime}(x)=\frac{\lambda_{n} f\left(x, u_{n}(x), u_{n}^{\prime \prime}(x)\right)}{\left\|u_{n}\right\|_{X}}, \quad x \in(0,1) \\
\omega_{n}(0)=\omega_{n}(1)=\omega_{n}^{\prime \prime}(0)=\omega_{n}^{\prime \prime}(1)=0
\end{array}\right.
$$

Similarly to (2.6), $\frac{\lambda_{n} f\left(x, u_{n}(x), u_{n}^{\prime \prime}(x)\right)}{\left\|u_{n}\right\|_{X}}$ is bounded, and thus $\left\{\omega_{n}^{\prime \prime \prime \prime}\right\}$ is bounded. By the AscoliArzelà theorem, choosing a subsequence and relabeling if necessary, it follows that there exists $(\widehat{\lambda}, \widehat{u}) \in\left[0, c_{0}\right] \times P$ with $\|\widehat{u}\|_{X}=1$ such that

$$
\lim _{n \rightarrow \infty}\left(\lambda_{n}, \omega_{n}\right)=(\widehat{\lambda}, \widehat{u}) \quad \text { in }\left[0, c_{0}\right] \times P
$$

Let

$$
\widetilde{f}(r)=\max \{f(x, s, p)|0 \leq|(s, p) \mid \leq r, x \in[0,1]\} .
$$

Then $\tilde{f}$ is nondecreasing, and (H3) implies that

$$
\lim _{r \rightarrow \infty} \frac{\tilde{f}(r)}{r}=0
$$


By (2.3) we have

$$
\frac{f\left(x, u_{n}(x), u_{n}^{\prime \prime}(x)\right)}{\left\|u_{n}\right\|_{X}} \leq \frac{\widetilde{f}\left(\sqrt{\left\|u_{n}\right\|_{\infty}^{2}+\left\|u_{n}^{\prime \prime}\right\|_{\infty}^{2}}\right)}{\left\|u_{n}\right\|_{X}} \leq \frac{\tilde{f}\left(\sqrt{2}\left\|u_{n}\right\|_{X}\right)}{\left\|u_{n}\right\|_{X}},
$$

which, together with (3.6) and $\left\|u_{n}\right\|_{X} \rightarrow \infty$, implies that

$$
\lim _{n \rightarrow \infty} \frac{f\left(x, u_{n}(x), u_{n}^{\prime \prime}(x)\right)}{\left\|u_{n}\right\|_{X}}=0 .
$$

Notice that (3.4) is equivalent to

$$
\omega_{n}(x)=\lambda_{n} \int_{0}^{1} G(x, s)\left[\int_{0}^{1} G(s, \tau) \frac{f\left(\tau, u_{n}(\tau), u_{n}^{\prime \prime}(\tau)\right)}{\left\|u_{n}\right\|_{X}} d \tau\right] d s, \quad x \in(0,1) .
$$

Combining this with (3.5) and using (3.7) and the Lebesgue dominated convergence theorem, it follows that

$$
\widehat{u}=\widehat{\lambda} \int_{0}^{1} G(x, s)\left[\int_{0}^{1} G(s, \tau) 0 d \tau\right] d s=0, \quad x \in(0,1) .
$$

This contradicts with $\|\widehat{u}\|_{X}=1$. Therefore, $\sup \{\lambda \mid(\lambda, u) \in \mathcal{C}\}=\infty$.

Step 2 . We show that $\sup \left\{\|u\|_{X} \mid(\lambda, u) \in \mathcal{C}\right\}=\infty$.

Assume on the contrary that $\sup \left\{\|u\|_{X} \mid(\lambda, u) \in \mathcal{C}\right\}=: M_{0}<\infty$. Let $\left\{\left(\lambda_{n}, u_{n}\right)\right\} \subset \mathcal{C}$ be such that

$$
\lambda_{n} \rightarrow \infty, \quad\left\|u_{n}\right\|_{X} \leq M_{0} .
$$

Then, by (2.3), $\left\|u_{n}\right\|_{\infty} \leq M_{0},\left\|u_{n}^{\prime \prime}\right\|_{\infty} \leq M_{0}$.

Since $\left(\lambda_{n}, u_{n}\right) \in \mathcal{C}$, using (2.2) we have

$$
\begin{aligned}
u_{n}(x) & =\lambda_{n} \int_{0}^{1} G(x, s)\left[\int_{0}^{1} G(s, \tau) f\left(\tau, u_{n}(\tau), u_{n}^{\prime \prime}(\tau)\right) d \tau\right] d s \\
& \geq \lambda_{n} \int_{0}^{1} G(x, s)\left[\int_{\frac{1}{4}}^{\frac{3}{4}} G(s, \tau) f\left(\tau, u_{n}(\tau), u_{n}^{\prime \prime}(\tau)\right) d \tau\right] d s \\
& =\lambda_{n} \int_{0}^{1} G(x, s)\left[\int_{\frac{1}{4}}^{\frac{3}{4}} G(s, \tau) \frac{f\left(\tau, u_{n}(\tau), u_{n}^{\prime \prime}(\tau)\right)}{u_{n}(\tau)} u_{n}(\tau) d \tau\right] d s \\
& \geq \frac{1}{4}\left\|u_{n}\right\|_{\infty} \lambda_{n} \int_{0}^{1} G(x, s)\left[\int_{\frac{1}{4}}^{\frac{3}{4}} G(s, \tau) \frac{f\left(\tau, u_{n}(\tau), u_{n}^{\prime \prime}(\tau)\right)}{\left\|u_{n}\right\|_{X}} d \tau\right] d s,
\end{aligned}
$$

which yields that $\left\{\lambda_{n}\right\}$ is bounded. This gives a contradiction.

Proof of Theorem 1.1. Let $\mathcal{C}$ be as in Lemma 2.2. By Lemma 2.4, $\mathcal{C}$ is bifurcating from $\left(\lambda_{1}(a, b), 0\right)$ and goes rightward. By Lemma 3.2 there exists a sequence $\left\{\left(\lambda_{n}, u_{n}\right)\right\} \subset \mathcal{C}$ such that $\lambda_{n} \rightarrow \infty$ and $\left\|u_{n}\right\|_{X} \rightarrow \infty$. It is easy to see that $\left\|u_{n}\right\|_{X} \rightarrow \infty$ implies $\left\|u_{n}\right\|_{\infty} \rightarrow \infty$, and then there exists $\left(\lambda_{0}, u_{0}\right) \in \mathcal{C}$ such that $\left\|u_{0}\right\|_{\infty}=4 s_{0}$. Lemma 3.1 implies that $\lambda_{0}<\lambda_{1}(a, b)$. By Lemmas 2.4, 3.1, and 3.2, $\mathcal{C}$ passes through some points $\left(\lambda_{1}(a, b), v_{1}\right)$ and $\left(\lambda_{1}(a, b), v_{2}\right)$ 
with $\left\|v_{1}\right\|_{\infty}<4 s_{0}<\left\|v_{2}\right\|_{\infty}$, and there exist $\underline{\lambda}$ and $\bar{\lambda}$ that satisfy $0<\underline{\lambda}<\lambda_{1}(a, b)<\bar{\lambda}$ and both (i) and (ii):

(i) if $\lambda \in\left(\lambda_{1}(a, b), \bar{\lambda}\right]$, then there exist $u$ and $v$ such that $(\lambda, u),(\lambda, v) \in \mathcal{C}$ and $\|u\|_{\infty}<\|v\|_{\infty}<4 s_{0}$

(ii) if $\lambda \in\left(\underline{\lambda}, \lambda_{1}(a, b)\right]$, then there exist $u$ and $v$ such that $(\lambda, u),(\lambda, v) \in \mathcal{C}$ and $\|u\|_{\infty}<4 s_{0}<\|v\|_{\infty}$.

Define $\lambda^{*}=\sup \{\bar{\lambda}: \bar{\lambda}$ satisfies (i) $\}$ and $\lambda_{*}=\inf \{\underline{\lambda}: \underline{\lambda}$ satisfies (ii) $\}$. Then (1.1) has positive solutions $u_{\lambda_{*}}$ at $\lambda=\lambda_{*}$ and $u_{\lambda^{*}}$ at $\lambda=\lambda^{*}$, respectively. Clearly, the component curve $\widetilde{\mathcal{C}}$ turns to the left at $\left(\lambda^{*},\left\|u_{\lambda^{*}}\right\|_{\infty}\right)$ and to the right at $\left(\lambda_{*},\left\|u_{\lambda_{*}}\right\|_{\infty}\right)$, that is, $\mathcal{C}$ is an $S$-shaped component. This, together with Lemma 3.2, completes the proof of Theorem 1.1.

Competing interests

The authors declare that they have no competing interests.

\section{Authors' contributions}

RM completed the main study, carried out the results of this article, and drafted the paper. JW checked the proofs and verified the calculation. Both authors read and approved the final manuscript.

\section{Author details}

${ }^{1}$ Department of Mathematics, Northwest Normal University, Lanzhou, 730070, P.R. China. ${ }^{2}$ Department of Applied Mathematics, Lanzhou University of Technology, Lanzhou, P.R. China.

\section{Acknowledgements}

This work was supported by the NSFC (No. 11671322, No. 11626016).

Received: 17 July 2016 Accepted: 18 October 2016 Published online: 28 October 2016

\section{References}

1. Gupta, CP: Existence and uniqueness theorems for the bending of an elastic beam equation. Appl. Anal. 26(4), 289-304 (1988)

2. Lazer, AC, McKenna, PJ: Large-amplitude periodic oscillations in suspension bridges: some new connections with nonlinear analysis. SIAM Rev. 32, 537-578 (1990)

3. Usmani, RA: A uniqueness theorem for a boundary value problem. Proc. Am. Math. Soc. 77, $329-335$ (1979)

4. Yang, YS: Fourth-order two-point boundary value problems. Proc. Am. Math. Soc. 104, 175-180 (1988)

5. Del Pino, MA, Manásevich, RF: Existence for a fourth-order boundary value problem under a two-parameter nonresonance condition. Proc. Am. Math. Soc. 112, 81-86 (1991)

6. Cabada, A, Cid, JÁ, Sanchez, L: Positivity and lower and upper solutions for fourth order boundary value problems. Nonlinear Anal. 67, 1599-1612 (2007)

7. Grunau, HC, Sweers, G: Positivity properties of elliptic boundary value problems of higher order. Nonlinear Anal. 8 5251-5258 (1997)

8. Grunau, HC, Sweers, G: Classical solutions for some higher order semilinear elliptic equations under weak growth conditions. Nonlinear Anal. 8, 799-807 (1997)

9. Schröder, J: Operator Inequalities. Mathematics in Science and Engineering, vol. 147. Academic Press, Inc., New York-London (1980)

10. Agarwal, RP: On fourth-order boundary value problems arising in beam analysis. Differ. Integral Equ. 2, 91-110 (1989)

11. Ma, R, Wang, HY: On the existence of positive solutions of fourth-order ordinary differential equations. Appl. Anal. 59, 225-231 (1995)

12. Bai, ZB, Wang, HY: On positive solutions of some nonlinear fourth-order beam equations. J. Math. Anal. Appl. 270, 357-368 (2002)

13. Li, Y: Positive solutions of fourth-order boundary value problems with two parameters. J. Math. Anal. Appl. 281, 477-484 (2003)

14. Drábek, P, Holubová, G, Matas, A, Nečesal, P: Nonlinear models of suspension bridges: discussion of the results. Appl. Math. 48, 497-514 (2003)

15. Liu, B: Positive solutions of fourth order two-point boundary value problems. Appl. Math. Comput. 148, 407-420 (2004)

16. Yao, Q: Existence, multiplicity and infinite solvability of positive solutions to a nonlinear fourth-order periodic boundary value problem. Nonlinear Anal. TMA 63, 237-246 (2005)

17. Han, G, Li, F: Multiple solutions of some fourth-order boundary value problems. Nonlinear Anal. 66, 2591-2603 (2007)

18. Ma, R: Existence of positive solutions of a fourth-order boundary value problem. Appl. Math. Comput. 168, 1219-1231 (2005)

19. $\mathrm{Ma}, \mathrm{R}, \mathrm{GaO}, \mathrm{C}, \mathrm{Han}, \mathrm{X}$ : On linear and nonlinear fourth-order eigenvalue problems with indefinite weight. Nonlinear Anal. 74, 4186-4191 (2011)

20. Dai, GW, Han, XL: Global bifurcation and nodal solutions for fourth-order problems with sign-changing weight. Appl. Math. Comput. 219, 9399-9407 (2013)

21. Sim, I, Tanaka, S: Three positive solutions for one-dimensional $p$-Laplacian problem with sign-changing weight. Appl. Math. Lett. 49, 42-50 (2015) 
22. De Coster, C, Habets, P: The lower and upper solutions method for boundary value problems. In: Cañada, A, Drábek, P, Fonda, A (eds.) Handbook of Differential Equations: Ordinary Differential Equations (2004)

23. Han, G, Xu, Z: Multiple solutions of some nonlinear fourth-order beam equations. Nonlinear Anal. 68, 3646-3656 (2008)

Submit your manuscript to a SpringerOpen ${ }^{\circ}$ journal and benefit from:

- Convenient online submission

- Rigorous peer review

- Immediate publication on acceptance

- Open access: articles freely available online

- High visibility within the field

- Retaining the copyright to your article

Submit your next manuscript at $\boldsymbol{~ s p r i n g e r o p e n . c o m ~}$ 